\title{
Evolutionary Consequences of Social Isolation
}

Nathan W. Bailey ${ }^{1 *}$ and Allen J. Moore ${ }^{2}$

Social isolation has profound impacts. Most animal research focuses on negative phenotypic consequences of social isolation within individual lifetimes. Less is known about how it affects genetics, selection, and evolution over longer timescales, though ample indirect evidence suggests that it might. We advocate that evolutionary consequences of social isolation be tested more directly. We suggest that the 'index of social isolation', the mismatch between actual and optimal social interaction experienced by individuals within a population, may play a key role in releasing cryptic genetic variation, adaptation rates, diversification patterns and ecosystem-level processes. Evolutionary dynamics arising from social isolation could have significant impacts in applied settings such as conservation, animal breeding, control of biological invasions and evolutionary resilience to anthropogenic change.

Keywords: conservation, indirect genetic effects, invasion biology, loneliness, social selection, sociogenomics

\footnotetext{
${ }^{1}$ School of Biology, University of St Andrews, St Andrews, Fife KY16 9TH, UK

2 Department of Entomology, College of Agricultural and Environmental Sciences, The University of Georgia, Athens, GA 30602, USA

* Correspondence: nwb3@st-andrews.ac.uk (N.W. Bailey)
} 
3

The psychological, physical, and societal impacts of social isolation are major research interests [1,2]. Despite clear connections to components of fitness such as health and well-being, little is known about whether or how the experience of social isolation might change evolutionary dynamics at population, species or ecosystem levels. Increasingly, there are good reasons to suspect that it might, so here we articulate an evolutionary research agenda and reasons for more directly studying social isolation from an evolutionary perspective. A key question is whether viewing social isolation as a unitary phenomenon could provide additional insight beyond what is gained from existing approaches in social evolution.

Social interactions are ubiquitous if not frequent, even amongst animals not classically considered to be social. Nearly every animal has the potential to experience social isolation. Many studies examining its effects understandably focus on social species, such as humans [2], other primates [3], and eusocial arthropods [4], and the study of social isolation has gained most traction in human research in which a well-developed literature focuses on the sociological, psychological and medical significance of experiencing separation from others. Despite this, the experience of social isolation is also highly relevant in species that are classified as asocial [5]. Social isolation can reflect individual behaviour, such as might occur when a subordinate male in a social hierarchy terminates a detrimental social interaction. It can also reflect characteristics of the environment outside the immediate control of a focal individual that impede or mask signal transmission ('signal masking'), such as anthropogenic pollution, habitat disturbance or signal jamming [6], literal physical separation that might occur during dispersal or vicariance events [7], exclusion from a social group [8], loss of social signals [9], or decreased social connectivity during conservation reintroductions [10]. Its effects may also be sexspecific [11]. Social isolation thus depends on the availability, detectability, and perception of social stimuli in the environment. Box 1 discusses its variation across taxa and contexts, which can be complicated by a number of life history and environmental factors, and defines a standardized index of social isolation to facilitate evolutionary studies. 
Social isolation is often viewed as having pathological effects, perhaps reflecting the impact of Henry Harlow's classic studies [12]. Accordingly, the impact of social isolation, either for a protracted duration during an individual's lifespan or for transient periods, is most often viewed as having negative fitness consequences [13]. If the experience of social isolation by individuals within a population is predictable from generation to generation, then evolutionary responses to this condition may be expected. Hypotheses about the adaptive significance and evolutionary causes of social isolation are increasingly being tested in other animals where experimental manipulations can be made readily. Table 1 describes key examples. Despite such advances, investigations into the impacts of perceived isolation from conspecifics have mainly been limited to evaluating its effect on individuals within their lifetimes. Comparatively little is known about trans-generational, evolutionary consequences of social isolation, but the field of evolutionary biology is well-equipped to address this.

Multiple subfields of evolutionary biology have spent decades interrogating the evolutionary impact of variation in social environments, both from theoretical and empirical angles. The significance of conspecific interactions to the evolutionary dynamics of sexual organisms is nearly axiomatic; usually, there can be no sexual reproduction without social interaction at some point (though counterexamples do exist, for example in externally-fertilising organisms). Nevertheless, studies of social evolution have almost exclusively focused on the effects of variation among social environments, for example to test adaptive benefits of sociality [14], and conditions lacking social interactions in laboratory-based experimental work are often treated as negative controls [e.g. 15,16]. Such experimental designs have driven extensive insights into social evolution, and are commonly used in behavioural, genetic and evolutionary studies of cognitive function [17], conflict and aggression [18], parental care $[19,20]$, and other topics. From these observations, it seems plausible that the experience of asocial conditions should significantly change evolutionary dynamics, as well. Asocial environments are frequently atypical. Our suggestion therefore is that the standard experimental paradigm can be usefully inverted, enabling researchers to view social isolation as a potentially critical factor in the evolutionary dynamics of sexual organisms. 
A research framework examining the role of social environments in evolution that emphasizes the importance of asocial experience can focus attention on the possibility that, in some cases, the absence of a social environment might be the determining factor shaping evolutionary dynamics of a population, species or ecosystem. In a trivial sense, permanent social isolation precludes sexual reproduction. However, it is also clear that transient episodes of social isolation can impact traits with prominent fitness consequences, and it is important to note that social isolation can have benefits in some circumstances, for example when crowding increases stress or competition [21]. Despite progress examining how social isolation impacts the expression of phenotypes within an individual's lifetime, little is known about variation in social isolation and how it affects the genetics of, and selection on, such traits. We further explore these mechanisms below.

Evolutionary Consequences of Social Isolation

What is meant by an evolutionary consequence of social isolation? The question here is not whether selection has historically favoured or disfavoured individual genotypes that are susceptible to social isolation [22,23], or genotypes that tend to impose it upon others ('ostracism', cf. [8]); various scenarios have been proposed to explain how the perception of social isolation might evolve as an adaptation or by-product of selection for other functions [24]. Likewise, adaptationist arguments for a function of social isolation have been developed within the field of human evolutionary psychology [25], but their validity can be difficult to test and the findings not easily applied to other species. Instead, we suggest that phenotypic variation of a trait, for example mate choice, might be influenced by social isolation. This will necessarily influence the phenotypes exposed to selection and therefore potential evolution of the trait. In addition, we suggest that variation in the timing and/or extent of social isolation may itself reflect genetic variation and therefore evolve via indirect genetic effects [IGEs; 26]. Box 2 describes several recent case studies that have explicitly examined the genetics of social isolation.

The ability to self-impose social isolation may represent an important adaptation under certain circumstances and result in social selection exerted upon other group members. Researchers studying the ant species Temnothorax unifasciatus manipulated the manner in which colony workers died: either naturally, by $\mathrm{CO}_{2}$ exposure, or by an induced infection with the pathogenic fungus Metarhizium 
anisopliae [4]. In all cases, dying workers removed themselves from the nest and remained socially isolated until death, a behaviour that dramatically eliminated social contact with other nestmates [4]. Their isolation not only quarantined them from nest mates, providing an adaptive benefit that might be maintained through kin selection, but it also made them die faster [4]. Transient social isolation appears to mitigate some of the costs associated with group living in social species, such as increased risk of parasite or disease transmission [1], although this must be balanced against the benefits of social immunity [27]. In humans, the existence of ostracism behaviour itself has been suggested to provide a mechanism for reducing resource monopolisation by dominant individuals in strong social hierarchies, suggesting that the ability to socially exclude others can generate selection for traits promoting egalitarianism $[28,29]$.

Social isolation's multifarious, time- and context-dependent costs and benefits are likely to heavily influence its effects on evolutionary dynamics. However, for social isolation to exert a significant influence on the evolution of a population or species, one condition must be met: experience of social isolation by an individual or individuals within the population must either change the genetic variation available to the action of selection, or it must change the action of selection itself. Either of these can be accomplished in a number of ways, and Box 3 details several case studies. There is evidence that social isolation can change gene expression [30] and induce epigenetic modifications $[17,31]$. The experience of social isolation also clearly affects fitness traits, for example by altering: mate choice and reproductive success [18,32], immunity or disease state [13,33], endocrine profiles [34,35], cooperative predator detection [36], communication [37], social competence [38], and the microbiome [39]. In a number of species, social isolation increases the likelihood of same-sex sexual behaviour (e.g. the fruit fly Drosophila melanogaster [40], the guppy Poecilia reticulata [41], Hermann's tortoises Testudo hermanni [42], and the zebra finch Taeniopygia guttata [43]). If fitness effects of social isolation are distributed non-randomly with respect to genotypes in a population, this can further impose an altered regime of social selection.

The manner by which social isolation might change the genetic architecture of traits can be usefully investigated from two perspectives. The first is by considering social isolation as an environmental factor which causes the release of cryptic genetic variation [44]. Standing genetic variation may not contribute to phenotypic variation when the social environment is relatively stable across generations; such cryptic genetic variation is hidden from selection. However, social isolation abruptly induces an 
environmental change, which could cause the expression of this previously masked genetic variation. Release of cryptic variation after environmental perturbation is a well-known phenomenon [45]. Even if a typical social environment is restored, the imprint of phenotypic effects caused by isolation may persist and provide a new phenotypic substrate upon which selection can act. Secondly, different genotypes might respond differently to asocial environments; such gene-by-social environment interactions can alter evolutionary potential by changing the outcome of sexual selection and exerting indirect genetic effects $[46,47]$. Consistent with this, specific genetic mutations in a mouse model have been linked to variation in sensitivity to social isolation [23]. Combined with findings from Rhesus macaques that the tendency to experience social isolation is variable and repeatable in a more naturalistic setting [3], and field crickets (Teleogryllus oceanicus) which show crossing reaction norms for mate discrimination after experiencing social versus asocial rearing conditions [48], gene-by-social environment interactions coupled with the release of cryptic genetic variation suggests a plausible mechanism driving evolutionary consequences of social isolation, and a promising avenue for future research.

With respect to how social isolation might alter selection, the most intuitive route to such an outcome is through changes in social selection. When behavioural [49], neural [50], physiological [35], morphological [15], immunological [27] and other traits change after experiencing social isolation, how then do those phenotypic changes impart different selection on other individuals during subsequent social interactions? Theoretical quantitative genetic models of social selection have illustrated the link between social interactions, their genetic causes, and changes in social selection [51]. Experiments which manipulate social isolation and quantify the impact on later episodes of selection would be particularly useful for testing how social isolation impacts selection, and we describe approaches for studying the evolutionary consequences of social isolation in Box 3.

\section{Evidence that Social Isolation Impacts Evolution}

Several recent studies shed light on the evolutionary impact of social isolation. Most evidence that has accumulated for an evolutionary role of social isolation is indirect - for example, studies consistent with Kaneshiro's hypothesis suggest that the relative isolation of founding populations favours the evolution of relaxed mating preferences [7], or studies that reveal substantial fitness consequences of social isolation. Work characterising how the experience of asocial versus social 
conditions alters the shape of selection provides further indication of how social isolation affects the potential for evolutionary change.

A study of the field cricket Gryllus pennsylvanicus quantified how social experience changed the shape of multivariate sexual selection on male traits such as age, size and condition [52] (Figure 1).

Females that had been socially isolated during development exerted considerably less sexual selection on males compared to females that had developed in an environment with access to conspecifics. In this case, female social isolation so dramatically decreased the opportunity for sexual selection on males (variance in relative fitness, $/$ [53]) that it was undetectable: from $/=0.760$ after experiencing social conditions to $/=0.151$ after experiencing isolated conditions. This difference manifested as a "flattening" of the multivariate selection surface imposed by female choice. It is logical to predict that such a reduction in the opportunity for selection would translate to less rapid or less pronounced evolutionary change.

(A)

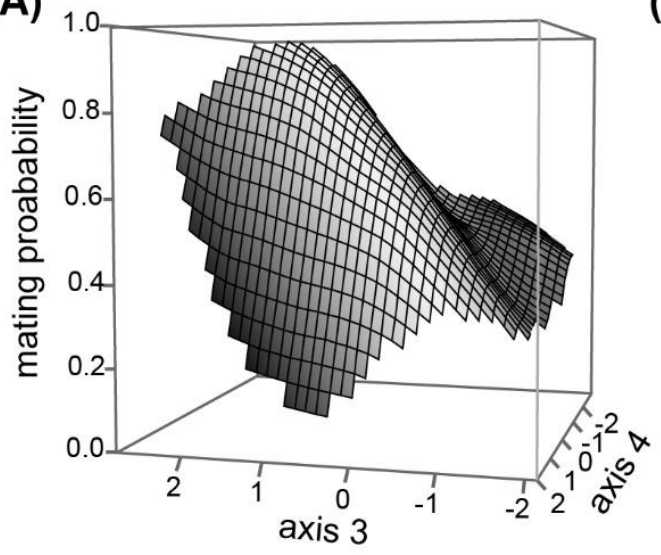

SOCIAL
(B)

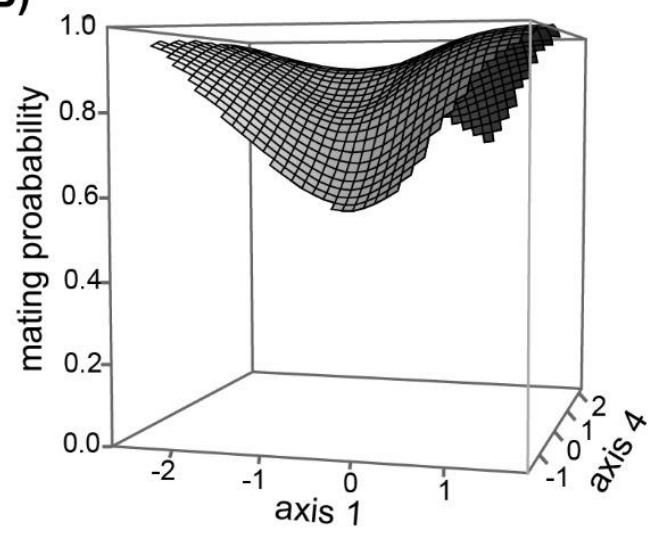

ASOCIAL

Figure 1. Multivariate fitness surfaces illustrating how female social isolation affects sexual selection on four male traits (head width, pronotum length, residual mass and mean age) in the cricket Gryllus pennsylvanicus [52]. The relationship between male mating probability ( $y$-axis) and two multivariate axes on which traits were loaded most heavily is shown for females that had been reared to adulthood in a mixed-sex social group (A) versus females that had experienced asocial conditions from their penultimate juvenile instar onward (B). Females reared in social conditions exerted strong and significant linear selection $\left(\theta_{i}=0.737\right)$ on axis 3 , for which male size attributes loaded heavily, whereas selection imposed by inexperienced females was weaker and quadratic $\left(\lambda_{i}=0.125\right)$. Additionally, experienced females exerted significant net selection favouring older males (standardised selection differential $s=0.210, p=0.034$ ), but net selection exerted by socially isolated females was undetectable (all $p>0.203$ ). Figure re-drawn with permission from [52]. 
182 Studies of a different insect, the burying beetle Nicrophorus vespilloides, have begun to document

183 such cross-generational responses to selection using laboratory populations experimentally evolved with or without parental care. Burying beetle larvae develop on dead mammal carcasses and are sometimes provisioned with food by their parents. The extent of this parental care varies in nature and across Nicrophorus species [54]. An experimental evolution study reared larvae with or without parents for seven generations and then split each experimental population into artificial selection treatments for small versus large size [55]. The researchers measured realized heritability of adult body size and responses to the artificially imposed selection regime and found that the heritability of size in beetles evolving without care was low, but only when under selection for large size (Figure 2) [55]. In contrast, social isolation enhanced responses to artificial selection for small size, confirming that the evolutionary effects of socially isolating conditions are likely to be context-dependent [55].

Work exploring the genetics of parenting behaviour in this system has probed the role of neuropeptide $\mathrm{F}$ (npf), a candidate gene with known functions in feeding. In N. vespilloides, expression of $n p f$ and its receptor, npf receptor, vary depending on the social experience of parents; isolated adults upregulate $n p f$ compared to adults exposed to larvae, in a way that could control the appropriate expression of feeding behaviour when larvae are present [56]. Social isolation also changes overall patterns of gene expression. When in the presence of their mate, males have little overlap with the gene expression of the parenting female and do not participate in parenting themselves [57]. If the female is absent, though, male gene expression is very similar to the female and he is an equally competent parent [57]. 


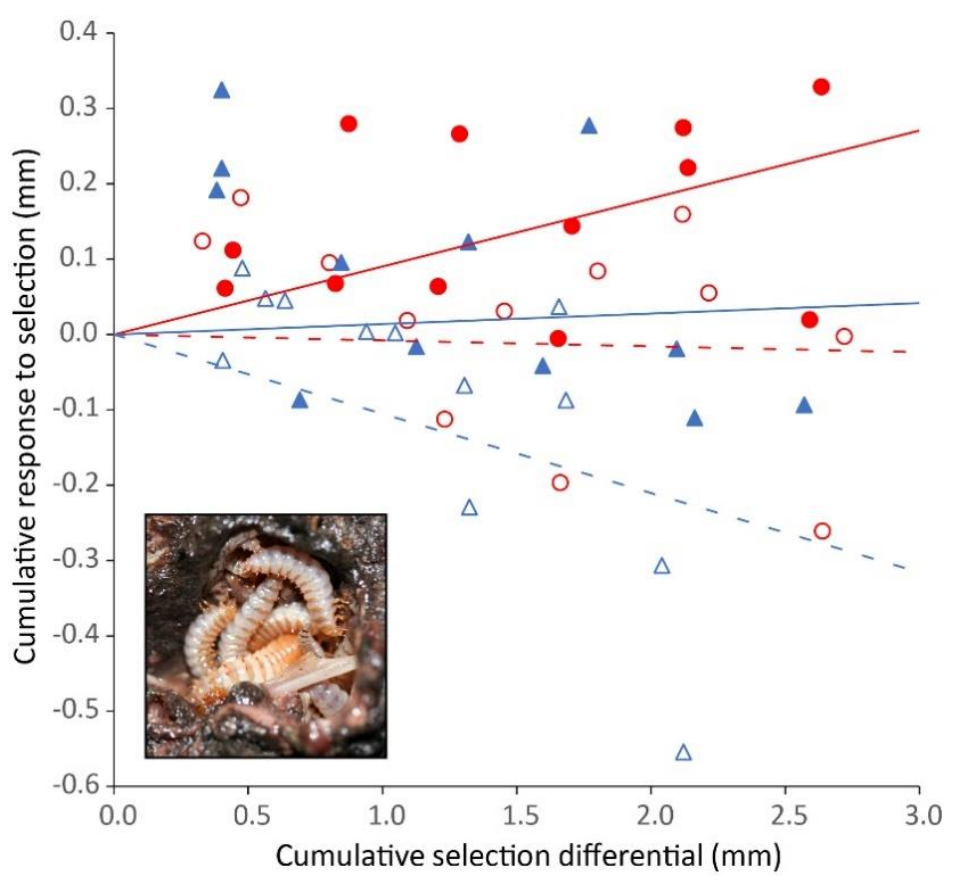

204

Figure 2. A specific form of social isolation (from parents) changes evolution in the burying beetle Nicrophorus vespilloides. An artificial evolution experiment examined realised heritability of body size in different social selection regimes over seven generations [55]. Artificial selection for large larval size (red symbols) was only effective when parental care was present (solid circles, solid line). Under this selective regime, persistent isolation from parents (open circles, dashed line) dampened the potential for evolution of body size. The opposite pattern was observed under an artificial selection regime for small larval size (blue symbols). Isolation from parents potentiated evolutionary responses for small size (open triangles, dashed line) whereas parental care dampened evolution of smaller size (solid triangles, solid line). Lines are regressions fitted to a zero intercept; their slope is the realised heritability of body size. The inset figure shows larvae feeding on a mouse carcass. (Graph redrawn with permission from [55]. Photo: Allen J. Moore).

In the burying beetle study, a lack of parental care does not equate to complete social isolation, because larvae developed together on carcasses even when parents were absent. However, persistent isolation from a crucial social interaction across multiple generations was shown to affect the genetics and response to selection of adult larval mass, a key fitness trait. A different experimental system has examined the impact of the evolutionary loss of a conspicuous sexual signal in the wild (Figure 3). In Hawaiian populations of the field cricket Teleogryllus oceanicus, singing males risk being infested by larvae of an acoustically-orienting endoparasitoid fly, Ormia ochracea. A male-silencing mutation called flatwing eliminates sound producing wing structures, protecting males from attack because the flies can no longer locate them [9]. Flatwing males were first detected around the turn of the last century and rapidly spread on multiple Hawaiian islands, and a key feature of this system is that the adaptive genetic variant causing flatwing eradicates the species' dominant long-range social signal - 
male song. In a population on the island of Kauai in particular, over 95\% of males are flatwing, a proportion that has remained stable for a decade [58], but which means that unless they happen to be in physical contact, crickets in this population effectively experience social isolation. Recent transcriptome profiling of the brains of crickets that carry genotypes for the normal, sound-producing wing type and crickets that carry genotypes for the male-silencing flatwing genotype found that crickets carrying genetic variants for flatwing are more sensitive to conditions of social isolation [30]. This genetic difference in susceptibility to social isolation appears to have coevolved with the rapidlyspreading genetic variant(s) that cause flatwing, and continuous behavioural monitoring of multiple Hawaiian populations suggests that compensatory responses to social isolation experienced by individuals developing on Kauai may have potentiated rapid adaptive evolution in this system [58].
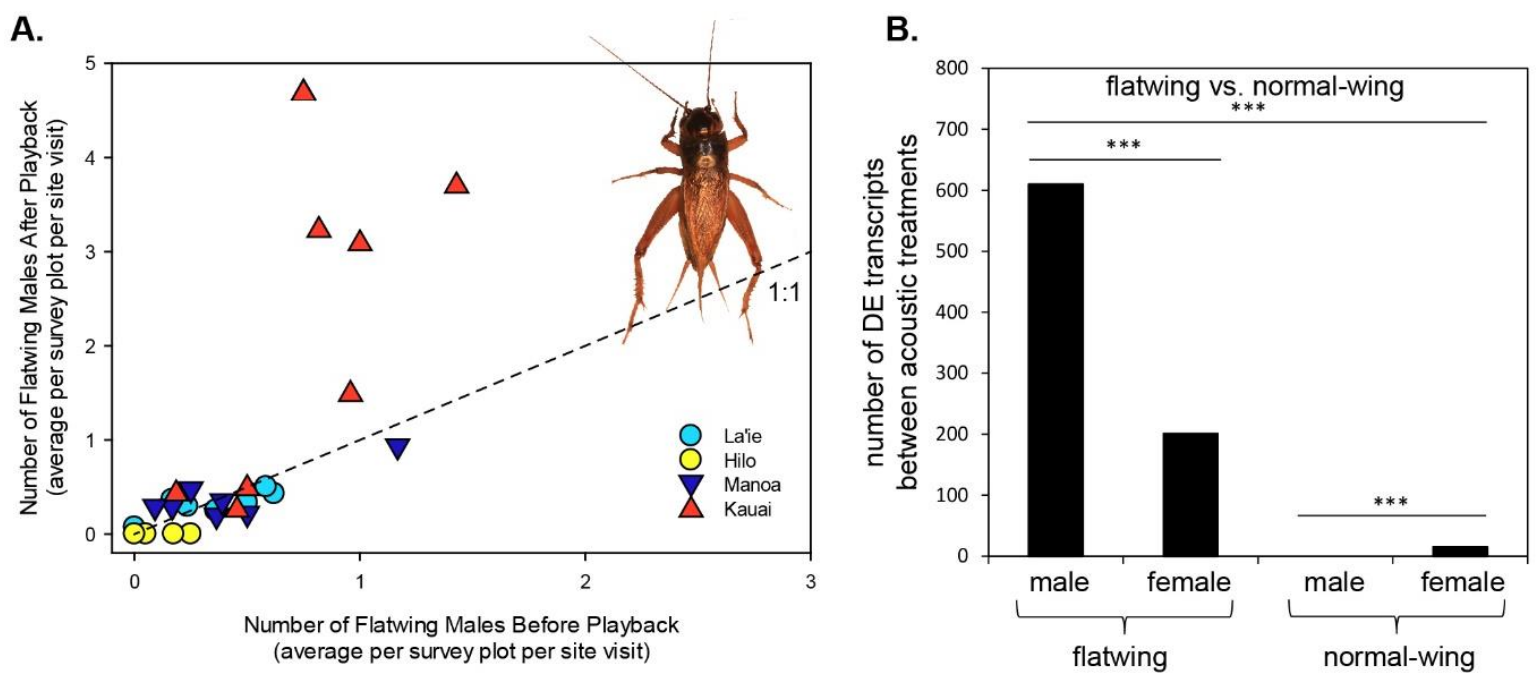

Figure 3. Behavioural and gene expression responses to social isolation in rapidly-evolving field crickets (Teleogryllus oceanicus). (A) A decade-long behavioural study compared the responsiveness of flatwing male crickets (pictured, inset) to acoustic playbacks in four Hawaiian populations on three islands [58]. The behavioural assay compared numbers of crickets in survey plots before and after artificially playing back islandspecific calling song. The graph shows the differential in numbers of crickets before vs. after the playback, with parity indicated by the dashed line. In the population on Kauai (red triangles), which has consistently contained nearly $100 \%$ silent flatwing males and thus a high perception of social isolation due to the lack of acoustic signalling, flatwing males are on average more likely to approach artificial playbacks. A similar pattern (not shown) is seen for females. (B) Crickets of both sexes carrying the flatwing genotype show a constitutive difference in their sensitivity to acoustic social signals in the environment for genes expressed in the brain [30]. Carriers of flatwing mutation(s) differentially express a greater number of genes when exposed to social isolation vs. a typical acoustic environment, consistent with the rapid coevolution of plasticity with adaptive genetic variants. (Graphs redrawn with permission from [58] and [30]. Photo: Nathan W. Bailey). 
254

255

256

257

258

259

260

261

262

263

264

265

266

267

268

269

270

271

272

Evolutionary dynamics that change as a result of social isolation might be particularly acute at the edges of range expansions, in initial founding propagules during biological invasions, or when anthropogenic activity fragments habitat. For example, the relative benefit of social feedback is enhanced at the extremes of an expanding range in the damselfly Ischneura elegans, promoting further range expansion [59]. Another intriguing example occurs in the invasive cane toad, Rhinella marina (Figure 4). In Australian populations at the leading edge of the toad invasion, there are few, but fast, individuals. Assortative mating has favoured long-range dispersers at the invasion front [60], but this in turn generates low-density populations and conditions of relative social isolation in newlyfounded habitats [61]. A study examining the effect of these factors used toads from a recentlycolonized ( $<3$ years) population in Western Australia to compare with toads from populations that had been evolving under relatively constant, high densities for approximately 80 years in eastern Australia and Hawaii [62]. Social attraction differed among the populations in a manner consistent with selection imposed by social isolation at the invasion front: toads of both sexes from the newlyestablished population in Western Australia were more likely to approach a social partner and spend more time with that individual. The authors concluded that increased social attraction at the range edge might beneficially increase information transfer and the likelihood of mating, suggesting that social isolation in such conditions might impose selection for adaptations to cope with it [62].

Figure 4. The effects of social isolation in invasive cane toads (Rhinella marina). The toads (inset photo) are exotic invaders in Hawaii and Australia. Colonies have persisted for many generations in Hawaii and Queensland, but the invasion front is comparatively new in Western Australia. Populations tend to be sparse at the invasion front, and a toads from a population at the invasion front exhibit more affiliative behaviours. Males' increased tendency to approach a fixed stimulus toad is illustrated. Such responses to social isolation, whether evolved or plastic, could have a positive impact on the success of invasive species. (Graph redrawn with permission from [62]. Photo: Nathan W. Bailey) 
290

291

292

293

294

295

Animal social interactions are simultaneously traits and environments. This duality has been recognized for over a century and a half, theoretically modelled as evolutionary feedback since the origins of the Modern Synthesis, and still forms the basis of lively modern debate about the adequacy of standard evolutionary theory. It is thus unsurprising that researchers have focused on the many ways in which social environments, and individual animals' sensitivity to variation in those environments, affect the evolutionary process [63]. We propose to capitalize on the theoretical and conceptual frameworks that have been developed as a result of this research activity, to study the ultimate causes and consequences of social isolation in animals. Useful frameworks for doing so include quantitative genetic theory on indirect genetic effects and gene-by-social environment interactions, in which a deficit of social interaction or asocial environments can be modeled and empirically manipulated. Neurogenetic approaches allow for the control and assessment of candidate genetic pathways that regulate the perception and reaction to social information in the environment. Large-scale experimental evolution studies can examine evolved responses to isolation from important social interactions such as parental care or sibling rivalry. And field studies of organisms for which dominant social signals have been lost or masked in nature enable researchers to assess the impact of social isolation in natural systems.

Why would such research activity be useful? What is the use of focusing on asocial conditions, when most of what defines animal life is its social structure? The fundamental dominance of social behaviour in evolutionary biology [64], and the preponderance of adaptations for, and unrelenting need to navigate, social interactions provides an answer to this question: to quote Mary Jane WestEberhard, "Individuals of social species having these specialized characteristics are in a sense trapped into group life, and group living may become virtually "obligatory" for them." [65, p.224]. Apart from providing data that can further inform the adaptive value of social isolation in different systems, understanding the mechanisms by which social isolation exerts evolutionary consequences can inform the processes underlying the evolutionary origins of group life to begin with. Moreover, understanding the evolutionary consequences of socially isolating animals will have relevance in applied contexts where such isolation may be an outcome of human activities, such as animal breeding, conservation reintroductions, efforts to control habitat loss and fragmentation in sensitive ecosystems, and adaptation to a changing environment. Multiple lines of evidence suggest that social 
isolation deserves serious evolutionary treatment. Some of its effects might be counter-intuitive, and researchers should be alert to the possibility that its fitness consequences will not always be negative $[66,21]$. We advocate grounding its study in rigorous, quantitatively-informed genetic frameworks. Hypothesis testing and manipulative experiments are essential. And finally, the observations we have outlined in this article hint that human society might be well-advised to reflect upon factors that contribute to our own social isolation, such as changes in the quality of social interaction driven by the proliferation of social media, and contemplate the long-term consequences of societal and cultural shifts in the way that we perceive and cope with social isolation.

Loneliness -

dangling from a nail,

a cricket.

\section{Matsuo Bashō (1644-1694) ${ }^{[67]}$}

\section{Acknowledgements}

We are grateful to Fiona C. Ingleby for insightful discussion about this topic and Lucas Marie-Orleach for helpful comments on a draft of the manuscript. Thanks are also due to the editor and three reviewers for thoughtful feedback and encouragement. N.W. Bailey was funded by the Natural Environment Research Council (NE/L011255/1) and A.J. Moore by the National Science Foundation (IOS-1354358).

\section{References}

1. Kappeler, P.M., et al. (2015) Sociality and health: impacts of sociality on disease susceptibility and transmission in animal and human societies. Phil. Trans. R. Soc. B 370, 20140116

2. Coplan, R.J. and Bowker, J.C. (Eds.) (2014) The handbook of solitude: psychological perspectives on social isolation, social withdrawl, and being alone. John Wiley \& Sons, Ltd, Chichester, UK.

3. Brent, L.J.N., et al. (2017) Persistent social isolation reflects identity and social context but not maternal effects or early environment. Sci. Rep. 7, 17791

4. Heinze, J. and Walter, B. (2010) Moribund ants leave their nests to die in social isolation. Curr. Biol. 20, 249-252

5. Lihoreau, M. et al. (2009) The weight of the clan: even in insects, social isolation can induce a behavioural syndrome. Behav. Process. 82:81-84

6. Barber, J.R., et al. (2010) The costs of chronic noise exposure for terrestrial organisms. Trends Ecol. Evol. 25, 180-189.

7. Tinghitella, R.M. and Zuk, M. (2009) Asymmetric mating preferences accommodated the rapid evolutionary loss of a sexual signal. Evolution 63, 2087-2098

8. Sasaki, T. and Uchida, S. (2013) The evolution of cooperation by social exclusion. Proc. R. Soc. B 280, 20122498

9. Zuk, M., et al. (2006) Silent night: adaptive disappearance of a sexual signal in a parasitized population of field crickets. Biol. Lett. 2, 521-524 
10. Teixeira, C.P. et al. (2007) Revisiting translocation and reintroduction programmes: the importance of considering stress. Anim. Behav. 73, 1-13

11. Leech, T., et al. (2017) Sex-specific effects of social isolation on ageing in Drosophila melanogaster. J. Insect. Physiol. 102:12-17

12. Harlow, H.F., et al. (1965) Total social isolation in monkeys. Proc. Natl Acad. Sci. USA. 54, 90-97

13. Hawkley, L.C. and Capitanio, J.P. (2015) Perceived social isolation, evolutionary fitness and health outcomes: a lifespan approach. Phil. Trans. R. Soc. Lond. B. 370, 20140114

14. Silk, J.B. (2007) The adaptive value of sociality in mammalian groups. Phil. Trans. R. Soc. Lond. B. 362, 539-559

15. Bailey, N.W. et al. (2010) Acoustic experience shapes alternative mating tactics and reproductive investment in field crickets. Curr. Biol. 20, 845-849

16. Han, C.S. and Brooks, R.C. (2014) Long-term effect of social interactions on behavioral plasticity and lifetime mating success. Am. Nat. 183, 431-444

17. Li, M. et al. (2016) Cognitive dysfunction and epigenetic alterations of the BDNF gene are induced by social isolation during early adolescence. Behav. Brain. Res. 313, 177-183

18. Kuriwada, T. (2016) Social isolation increases male aggression toward females in the field cricket Gryllus bimaculatus. Popul. Ecol. 58, 147-153

19. Banerjee, S.B., et al. (2012) Deprivation of maternal care has long-lasting consequences for the hypothalamic-pituitary-adrenal axis of zebra finches. Proc. R. Soc. B 279, 759-766

20. Nyman, C., et al. (2017) Effect of the early social environment on behavioural and genomic responses to a social challenge in a cooperatively breeding vertebrate. Molec. Ecol. 26, 3186-3203

21. Svensson, E., et al. (2001) Density-dependent competition and selection on immune function in genetic lizard morphs. Proc. Natl. Acad. Sci., USA 98, 12561-12565

22. Distel, M.A. et al. (2010) Familial resemblance for loneliness. Behav. Genet. 40, 480-494

23. Gresack, J. et al. (2010) $\mathrm{CRF}_{2}$ null mutation increases sensitivity to isolation rearing effects on locomotor activity in mice. Neuropeptides 44, 349-353

24. Cacioppo, J.T. et al. (2013) Evolutionary mechanisms for loneliness. Cogn. Emot. 28, 3-21

25. Keller, M.C. and Nesse, R.M. (2005) Is low mood an adaptation? Evidence for subtypes with symptoms that match precipitants. J. Affect. Disord. 86, 27-35

26. Moore, A.J. et al. (1997) Interacting phenotypes and the evolutionary process: I. Direct and Indirect Genetic Effects of Social Interactions. Evolution 51, 1352-1362

27. Rödel, H.G. and Starkloff, A. (2014) Social environment and weather during early life influence loads of an intestinal parasite in a group-living small mammal. Oecologia, 176, 389-398.

28. Boehm, C. (1999). Hierarchy in the forest: The Evolution of Egalitarian Behaviour. Harvard University Press. Cambridge, Massachusetts

29. Gavrilets, S., et al. (2008) Dynamics of alliance formation and the egalitarian revolution. PLoS ONE 3, e3293

30. Pascoal, S. et al. (2018) Increased socially-mediated plasticity in gene expression accompanies rapid adaptive evolution. Ecol. Lett. 21, 546-556.

31. Sasagawa, T., et al. (2017) Long-term effects of maternal separation coupled with social isolation on reward seeking and changes in dopamine D1 receptor expression in the nucleus accumbens via DNA methylation in mice. Neurosci. Lett. 641, 33-39

32. Uzsák, A. and Schal, C. (2013) Social interaction facilitates reproduction in male German cockroaches, Blatella germanica. Anim. Behav. 85, 1501-1509

33. Kohlmeier, P. et al. (2016) Survival after pathogen exposure in group-living insects: don't forget the stress of social isolation! J. Evol. Biol. 29, 1867-1872

34. Cacioppo, J.T., et al. (2015) The neuroendocrinology of social isolation. Annu. Rev. Psychol. 66, 733-767

35. Galhardo, L. and Oliveira, R.F. (2014) The effects of social isolation on steroid hormone levels are modulated by previous social status and context in a cichlid fish. Horm. Behav. 65, 1-5

36. Hesse, S. et al. (2015) Social deprivation affects cooperative predator inspection in a cichlid fish. $R$. Soc. Open Sci. 2, 140451 
37. Keesom, S.M., et al. (2017). Early-life social isolation influences mouse ultrasonic vocalizations during male-male social encounters. PLOS ONE 12, e0169705

38. Lesne, P., et al. (2016) Early experience and social performance in spiderlings. Anim. Behav. 122, 9-16

39. Ntranos, A. and Casaccia, P. (2018) The microbiome-gut-behavior axis: crosstalk between the gut microbiome and oligodentrocytes modulates behavioral responses. Neurotherapeutics 15, 31-35

40. Bailey, N.W., et al. (2013) Measuring same-sex sexual behaviour: the influence of the male social environment. Anim. Behav. 86, 91-100

41. Field, K.L. and Waite, T.A. (2004) Absence of female conspecifics induces homosexual behaviour in male guppies. Anim. Behav. 68, 1381-1389

42. Bonnet, $X_{\text {., }}$ et al. (2016) A prison effect in a wild population: a scarcity of females induces homosexual behaviors in males. Behav. Ecol. 27, 1206-1215

43. Adkins-Regan, E. (2002) Development of sexual partner preference in the zebra finch: a socially monogamous, pair-bonding animal. Arch. Sex. Behav. 31, 27-33

44. Paaby, A.B. and Rockman, M.V. (2014). Cryptic genetic variation: evolution's hidden substrate. Nat. Rev. Genet. 15, 247-258

45. Gibson, G. and Dworkin, I. (2004) Uncovering cryptic genetic variation. Nat. Rev. Genet. 5, 681-690

46. Ingleby, F.C., et al. (2010) The role of genotype-by-environment interactions in sexual selection. J. Evol. Biol. 23, 2031-2045

47. Wolf, J.B., et al. (2014) Genotype-by-environment interactions when the social environment contains genes. In: Genotype-by-Environment Interactions and Sexual Selection (Eds. J. Hunt and D. Hosken). John Wiley \& Sons, Ltd, Chichester, UK

48. Bailey, N.W. and Zuk, M. (2012) Socially flexible female choice differs among populations of the Pacific field cricket: geographical variation in the interaction coefficient psi $(\Psi)$. Proc. R. Soc. Lond. B. 279, 35893596

49. McCarthy, K., et al. (2015) The effect of social isolation on locomotor activity in the houseflies (Musca domestica). J. Insect Behav. 28, 288-296

50. Silva-Gómez, A.B., et al. (2003) Decreased dendritic spine density on prefrontal cortical and hippocampal pyramidal neurons in postweaning social isolation rats. Brain Res. 983, 128-136

51. McGlothlin, J.W. et al. (2010) Interacting phenotypes and the evolutionary process. III. Social evolution. Evolution 64, 2558-2574

52. Judge, K.A. (2010) Female social experience affects the shape of sexual selection on males. Evol. Ecol. Res. 12, 389-402

53. Arnold, S.J. and Wade, M.J. (1984) On the measurement of natural and sexual selection: theory. Evolution 38, 709-719

54. Benowitz, K.M., et al. (2016) Difference in parenting in two species of burying beetle, Nicrophorus orbicollis and Nicrophorus vespilloides. J. Ethol. 34, 315-319

55. Jarrett, B.J.M., et al. (2017) Cooperative interactions within the family enhance the capacity for evolutionary change in body size. Nat. Ecol. Evol. 1, 0178

56. Cunningham, C.B., et al. (2016) The role of neuropeptide F in a transition to parental care. Biol. Lett. 12, 20160158

57. Parker, D.J., et al. (2015) Transcriptomes of parents identify parenting strategies and sexual conflict in a subsocial beetle. Nat. Comm. 6:8449

58. Zuk, M., et al. (2018) Sexual signal loss: the link between behavior and rapid evolutionary dynamics in a field cricket. J. Anim. Ecol. 87, 623-633

59. Lancaster, L.T., et al. (2017) Do group dynamics affect colour morph clines during a range shift? J. Evol. Biol. 30, 726-737.

60. Phillips, B.L., et al. (2006) Invasion and the evolution of speed in toads. Nature 439, 803

61. Phillips, B.L., et al. (2010) Evolutionarily accelerated invasions: the rate of dispersal evolves upwards during the range advance of cane toads. J. Evol. Biol. 23, 2595-2601

62. Gruber, J., et al. (2017) The loneliness of the long-distance toad: invasion history and social attraction in cane toads (Rhinella marina). Biol. Lett. 13, 20170445 
63. West-Eberhard, M.J. (2014) Darwin's forgotten idea: The social essence of sexual seletion. Neurosci. Behav. Rev. 46, 501-508

64. Alexander, R.D. (1974) The evolution of social behavior. Annu. Rev. Ecol. Syst. 5, 325-383

65. West-Eberhard, M.J. (1979) Sexual selection, social competition and evolution. Proc. Am. Philos. Soc. $123,222-234$

66. Blumstein, D.T., et al. (2018) Strong social relationships are associated with decreased longevity in a facultatively social mammal. Proc. R. Soc. Lond. B. 285:20171934

67. Barnhill, D.L. (2004) Bashō's Haiku: Selected Poems of Matsuo Bashō. SUNY Press, Albany, USA

68. Bailey, N.W. (2011) Mate choice plasticity in the field cricket Teleogryllus oceanicus: effects of social experience in multiple modalities. Behav. Ecol. 65, 2269-2278

69. Schausberger, P. et al. (2017) Early social isolation impairs development, mate choice and grouping behaviour of predatory mites. Anim. Behav. 127, 15-21

70. Perret, A. et al. (2015) Social visual contact, a primary "drive" for social animals? Anim. Cogn. 18, 657-666

71. Stoffer, B. and Uetz, G.W. (2017) The effects of experience with different courtship modalities on unimodal and multimodal preferences in a wolf spider. Anim. Behav. 123, 187-196

72. Verzijden, M.N. and Rosenthal, G.G. (2011) Effects of sensory modality on learned mate preferences in female swordtails. Anim. Behav. 82, 557-562

73. Lenoir, A., et al. (2001) Effects of social isolation on hydrocarbon pattern and nestmate recognition in the ant Aphaenogaster senilis (Hymenoptera, Formicidae). Insectes Soc. 48, 101-109

74. Niwa, M., et al. (2016) A critical period of vulnerability to adolescent stress: epigenetic mediators in mesocortical dopaminergic neuron. Hum. Molec. Genet. 25, 1370-1381

75. Gao, J. et al. (2017) Genome-wide association study of loneliness demonstrates a role for common variation. Neuropsychopharmacol. 41, 811-821

76. Drown, D.M. and Wade, M.J. (2014) Runaway coevolution: adaptation to heritable and nonheritable environments. Evolution 68, 3039-3046

77. Bailey, N.W., et al. (2018) Indirect genetic effects in behavioral ecology: does behavior play a special role in evolution? Behav. Ecol. 29, 1-11

78. Kurvers, R.H.J.M, et al. (2014) The evolutionary and ecological consequences of animal social networks: emerging issues. Trends Ecol. Evol. 29, 326-335

79. Crudgington, H.S., et al. (2009) Experimental manipulation of sexual seletion promotes greater male mating capacity but does not alter sperm investment. Evolution 63, 926-938

80. Crudgington, H.S., et al. (2005) Experimental removal and elevation of sexual selection: does sexual selection generate manipulative males and resistant females? Am. Nat. 165, S72-S87

81. Snook, R.R., et al. (2005) Experimental manipulation of sexual selection and the evolution of courtship song in Drosophila pseudoobscura. Behav. Genet. 35, 245-255

82. Veltsos, P., et al. (2017) Mating system manipulation and the evolution of sex-biased gene expression in Drosophila. Nat. Comm. 8, 2072

83. Willink, B. and Svensson, E.I. (2017) Morphs, dispersal behavior, genetic similarity, and the evolution of cooperation. Proc. R. Soc. Lond. B. 284, 20162407

84. Robinson, G.E., et al. (2005) Sociogenomics: social life in molecular terms. Nat. Rev. Genet. 6, 257-270

85. Taborsky, B. and Oliviera, R.F. (2012) Social competence: an evolutionary approach. Trends Ecol. Evol. 27, 679-688 
Box 1. What is Social Isolation? Perception versus Reality

Is social isolation simply a physical separation from other individuals which blocks all social sensory information in the environment? Social stimuli such as visual, olfactory, acoustic and tactile cues are both emitted and received by individual animals. The experience of social isolation by a focal organism therefore depends only partly on actual separation from conspecifics; the experience of social isolation and its attendant phenotypic effects can also arise from the inability to produce or receive such stimuli [35]. In addition, different modalities and types of social stimuli might not be equally important [68], and the timing of periods of isolation across the life course can determine its phenotypic outcome $[69,38,49]$. We suggest that social isolation can be measured phenotypically as an individual's deviation from a population-level optimum. Assuming weak stabilizing selection and evolutionary equilibrium, the optimum level of social interaction is estimated as the population mean. Social isolation can then be measured as the signed deviation from the mean for each individual, where negative values represent a deficit of social interaction: i.e. an individual's index of social isolation. The index of social interaction is thus a quantitative measure and allows for quantitative genetic treatment, examination of threshold dynamics, and standardized comparison across groups, populations or taxa in evolutionary studies. The index of social isolation accounts for the fact that what drives the experience of social isolation will vary widely across contexts, and might unevenly impact different fitness traits [11].

In the European starling Sturnus vulgaris for example, visual contact with conspecifics has been suggested to be a basic need for appropriate functioning, or social competence, and the requirement for social visual contact may even be primary to non-social needs [70]. Researchers tested a similar idea in wolf spiders (Schizocosa ocreata) by manipulating juvenile females' experience of male sexual signals [71]. Male signals occur in different modalities: they produce percussive vibratory signals transmitted through substrate, and visual signals arising from dark bristles on their legs. A lack of vibratory signals experienced during development decreased adult receptivity to vibratory signals during later no-choice trials; adult receptivity in the visual channel was not impaired to the same extent by a lack of juvenile visual experience [71]. Despite this, females preferentially responded to multimodal signals regardless of their prior experience [71]. Mate choice in swordtails also depends on both visual and olfactory signals. Females' preferences develop through exposure to conspecifics, but the timing of exposure and development differs for the two modalities [72]. 
The timing and duration of social isolation can also change its phenotypic outcome. In the ant Aphaenogaster senilis, hydrocarbons function as important social signals that control nestmate recognition and are repeatedly exchanged among individuals during physical encounters. This social feedback plays a key role in the maintenance of the hydrocarbon profiles of individual ants, and social isolation causes the profiles to change rapidly [73]. With progressively longer durations of social

552 isolation, ants' hydrocarbon profiles changed more extensively, with corresponding increases in the

553 likelihood that they would be attacked by former nestmates upon reintroduction [73].

Using a transgenic mouse model, researchers evaluated the effect of psychosocial stress during adolescence by imposing three weeks of social isolation during this critical developmental period [74]. In this study, group-housing acted as the control condition. The researchers found that social

558 isolation epigenetically modified the gene tyrosine hydroxylase, disrupting glucocorticoid signaling.

559 Through a series of experimental treatments, they isolated this adverse neurogenetic effect of social

560 isolation to the first week, and only the first week, of adolescence, identifying a key developmental 561 window during which social isolation exerts a significant phenotypic impact [74]. Despite relatively 562 brief developmental timeframes for the experience of social isolation to exert a phenotypic influence 563 on focal individuals, the effects of even transient exposure to asocial conditions can persist 564 throughout an individual's lifetime $[31,69]$. 
Box 2. Genetics of Social Isolation

Recent studies have sought to characterize the genetic basis of perceived social isolation, for example by partitioning genetic variance underlying familial resemblance for loneliness in humans [22]. A recent genome-wide association study was unable to identify causal variants, despite evidence for moderate heritability of loneliness in humans [75]. A study of Rhesus macaques (Macaca mulatta) used a social network approach on a dataset that spanned approximately half of an adult lifespan, uncovering repeatable differences in several measures of the degree to which individuals were socially isolated [3]. These repeatable differences in social isolation suggest the potential for underlying genetic effects, but may also arise from general social network stability.

Neurogenetic studies in model organisms have addressed questions about social isolation's impacts by examining candidate genes with suspected functions in social behaviours. A study of rats examined the effect of social isolation on brain-derived neurotrophic factor (BDNF) expression, which has been related to cognitive functioning [17]. Adolescent rats were subjected to two weeks of either social isolation or group conditions with two conspecifics. Following the experimental treatment, all rats were resocialized. Those that had experienced social isolation later showed impaired prepulse inhibition, i.e. a decreased neural capacity to process external stimuli without interruption [17]. This neural impairment was associated with acetylation modifications to the BDNF gene and corresponding changes in BDNF expression in the medial prefrontal cortex and hippocampus of isolated rats' brains [17]. In mice, epigenetic gene regulation also controls changes in the expression of neural dopamine receptors following social isolation, suggesting functional genetic mechanisms involving dopaminergic neurotransmission and the potential for long-term, trans-generational phenotypic responses to social isolation [31] (Figure I).

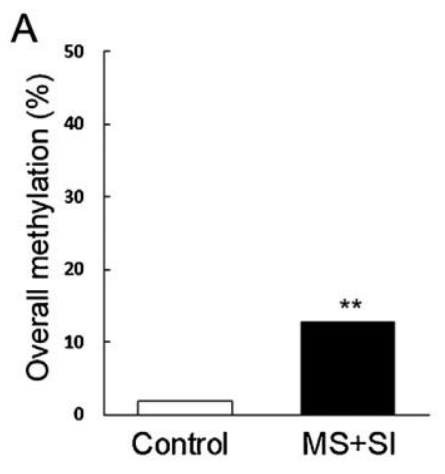

B

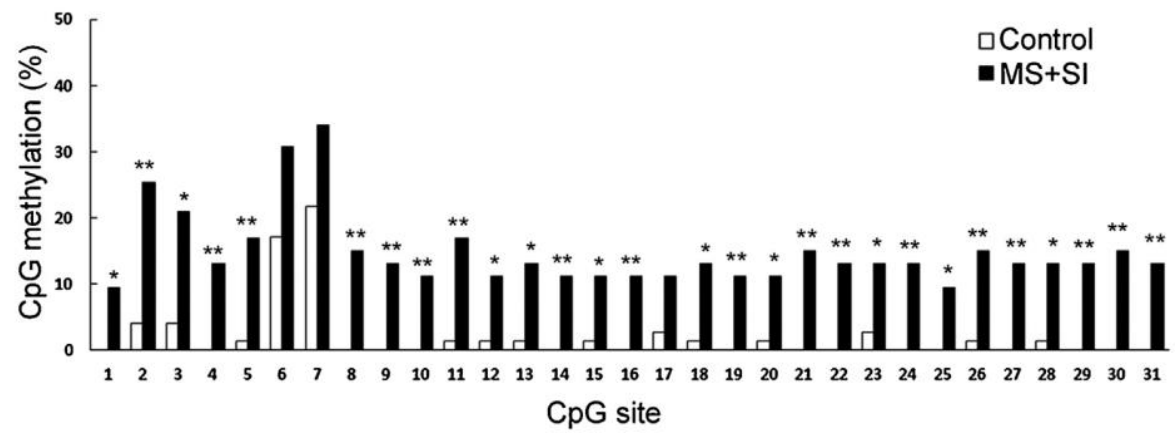


Figure I. Social isolation causes gene expression changes via epigenetic modification in mice [31]. Newborn

594 mouse pups were separated from maternal contact and from contact with other pups for three hours on a daily

595 basis for two weeks. In females, the experience of social isolation caused decreased food seeking behaviour,

596 which was consistent with a change in dopaminergic reward systems of the brain. The involvement of brain

597 dopamine circuits was supported by the observation that social isolation reduced both mRNA and protein

598 expression levels of a dopamine receptor gene (Drd7a) in the nucleus acumbens. This downregulation appears

599 to be caused by hypermethylation of the Drd7a gene in socially isolated females (MS+SI), shown in (A) above.

600 (B) Methylation status was assessed at 31 sites within Drd7a (CpG sites in figure above); significant differences in

601 methylation status were found in 29 of these, with all cases indicative of hypermethylation following social

602 isolation. Figure reproduced with permission from [31]. 
604

605

606

607

608

609

610

611

612

613

614

615

616

617

618

619

620

621

622

623

624

625

626

627

628

629

630

631

632

633

634

635

Box 3. Approaches for Testing the Evolutionary Role of Social Isolation

Clearly identified hypotheses are essential for investigating social isolation from an evolutionary perspective $[26,51,76]$ (Table 1), and several well-established frameworks could be used to test them. For example, variance partitioning approaches using interacting phenotype theory can quantify the impact of variation in the social environment on additive genetic variation, heritability, and opportunity for selection [26]. If the genetic background of interacting partners is experimentally manipulated, IGEs exerted by interacting partners that have experienced social versus asocial environments can be quantified and compared, allowing a test of whether social isolation is likely to potentiate or stymie evolutionary change [77]. Calculation and comparison of selection coefficients using standard regression-based techniques provides an additional dimension of information [53], and there is similarity to testing the evolutionary consequences of social networks [78].

Quantifying the effects of social isolation on genetics and selection is informative, but the insights gained are mostly limited to how social isolation affects evolutionary potential, as opposed to realised evolutionary change. Experimental evolution approaches provide a powerful means for manipulating and observing those sorts of evolutionary changes. Experimental evolution lines of the fruit fly Drosophila pseudoobscura provide an instructive example. Lines have been maintained for over 100 generations under different mating system regimes; while not reflecting any period of absolute social isolation per se, the monogamous treatment pairs one female with one male, while females evolved under polyandrous conditions have access to either 3 or 6 males [79]. Experimental removal of sexual selection has been found to drive evolutionary changes in male mating investment [79], female fecundity [80], male courtship song [81] and gene expression [82]. While these social manipulations were performed to test the effects of mating system, they provide a blueprint for how populations that contain individuals with different indices of social isolation could be experimentally evolved. Many species might not be amenable to such laboratory-based experimental evolution approaches, but the existence of segregating marker phenotypes, such as flatwing morphology in the cricket example above, or discrete colour morphs in the damselfly Ischneura elegans [83], could be used to measure evolutionary responses after manipulating social isolation of different morphs. Comparative work examining natural populations or taxa that vary in their degree of social isolation would also help to validate experimental findings against observations from natural systems, and this can be coupled with sociogenomics approaches [84] to dissect the underlying genetics. 


\begin{tabular}{|c|c|c|c|}
\hline Hypothesis & Prediction & Example & Refs \\
\hline
\end{tabular}

\section{EVOLUTIONARY CAUSES}

\begin{tabular}{llll}
\hline Self-Quarantine & $\begin{array}{l}\text { Social isolation prevents } \\
\text { disease and pathogen } \\
\text { transmission to kin }\end{array}$ & $\begin{array}{l}\text { Social isolation is maintained by kin selection as a colony-level } \\
\text { defense against pathogen transmission in the ant Temnothorax } \\
\text { unifasciatus. }\end{array}$ & {$[4]$} \\
\hline Ostracism & $\begin{array}{l}\text { Social exclusion of free-riding } \\
\text { individuals protects enforcers } \\
\text { against exploitation }\end{array}$ & $\begin{array}{l}\text { Game-theoretic models found that ostracism of costly free- } \\
\text { riders can be a stable strategy which reduces the costs } \\
\text { associated with punishing defectors. }\end{array}$ & {$[8]$} \\
\hline Social & $\begin{array}{l}\text { Individuals that threaten others } \\
\text { Manipulation }\end{array}$ & $\begin{array}{l}\text { Related to the concept of ostracism above. The threat of social } \\
\text { with social isolation gain fitness }\end{array}$ & $\begin{array}{l}\text { isolation may be an effective means of controlling other } \\
\text { individuals, favoring genotypes that can use and follow through } \\
\text { benefits }\end{array}$ \\
& such threats. & \\
\hline
\end{tabular}

\section{EVOLUTIONARY CONSEQUENCES}

\begin{tabular}{llll}
\hline $\begin{array}{l}\text { Potentiates } \\
\text { evolution }\end{array}$ & $\begin{array}{l}\text { Social isolation exposes cryptic } \\
\text { genetic variation to the action } \\
\text { of selection }\end{array}$ & $\begin{array}{l}\text { Field crickets (Teleogryllus oceanicus) that lost their dominant } \\
\text { social signal, male song, perceive an asocial environment. } \\
\text { However, they respond flexibly to this, enabling a rapid } \\
\text { evolutionary response. }\end{array}$ & [30] \\
\hline $\begin{array}{llll}\text { Inhibits } \\
\text { evolution }\end{array}$ & $\begin{array}{l}\text { Experience of social isolation } \\
\text { reduces the opportunity for } \\
\text { selection }\end{array}$ & $\begin{array}{l}\text { Previous experience of social isolation in female crickets, Gryllus } \\
\text { pennsylvanicus, reduced the amount of sexual selection they } \\
\text { later exerted on males. }\end{array}$ & [52] \\
\hline $\begin{array}{l}\text { Promotes } \\
\text { evolution of } \\
\text { cooperation }\end{array}$ & $\begin{array}{l}\text { Social isolation selects for } \\
\text { prosocial behaviours that } \\
\text { mitigate isolation's negative } \\
\text { fitness effects }\end{array}$ & $\begin{array}{l}\text { Experimental evolution studies in Nicrophorus vespilloides show } \\
\text { that consistent isolation from parental care drives higher levels }\end{array}$ & [55] \\
\hline $\begin{array}{l}\text { Drives evolution larval cooperation. } \\
\text { of greater } \\
\text { social affinity }\end{array}$ & $\begin{array}{l}\text { As above, social isolation } \\
\text { selects for prosocial behaviours } \\
\text { that mitigate isolation's }\end{array}$ & $\begin{array}{l}\text { Comparatively isolated cane toads (Rhinella marina) at an } \\
\text { invasion front exhibit increased social attraction compared to }\end{array}$ & [62] \\
those from long-established, denser populations. & \\
\hline
\end{tabular}

638

639 
Cryptic genetic variation: genetic variation that remains unexpressed at the level of observable phenotypes, unless environmental circumstances change.

Gene-by-(social) environment interaction: when the genotypic contribution to trait variation differs across environments.

Index of social isolation: mismatch between the frequency and quality of social interaction required to optimise fitness, and an individual's experienced frequency and quality of social interactions.

Indirect genetic effects: when genes expressed in social partners alter trait expression in focal individuals.

Loneliness: in humans, a mismatch between required levels of social interaction and perceived levels available in the social environment [13] (contrast with 'index of social isolation').

Ostracism: exclusion of individuals through the coordinated action of a larger group [8].

Signal masking: when the detection threshold for a signal is increased because of the presence of other signals (noise) in the environment

Social competence: the intrinsic ability of an individual to optimize their social behaviour to their social environment [85].

Social immunity: group-level defenses against infection which benefit individuals.

Social selection: selection arising when individual fitness is affected by social competition with conspecifics [65].

Sociogenomics: an integrated approach using techniques such as genome-sequencing, gene expression profiling, proteomics, and behavioural assays to understand the genetic and 